\title{
Hepatic Encephalopathy Expands the Predictivity of Model for End-Stage Liver Disease in Liver Transplant Setting: Evidence by Means of 2 Independent Cohorts
}

Cristina Lucidi, ${ }^{1}$ Stefano Ginanni Corradini, ${ }^{1}$ Juan G. Abraldes, ${ }^{3}$ Manuela Merli, ${ }^{1}$ Puneeta Tandon, ${ }^{3}$ Flaminia Ferri, ${ }^{1}$ Lucia Parlati, ${ }^{1}$ Barbara Lattanzi, ${ }^{1}$ Edoardo Poli, ${ }^{1}$ Vincenza Di Gregorio, ${ }^{1}$ Alessio Farcomeni, ${ }^{2}$ and Oliviero Riggio ${ }^{1}$

${ }^{1}$ Division of Gastroenterology, Department of Clinical Medicine, University of Rome, Rome, Italy; ${ }^{2}$ Public Health and Infectious Diseases, "Sapienza" University of Rome, Rome, Italy; ${ }^{3}$ Cirrhosis Care Clinic, Liver Unit, Division of Gastroenterology, University of Alberta, Edmonton, Canada

\begin{abstract}
Despite its documented prognostic relevance, hepatic encephalopathy (HE) is not considered in liver transplantation (LT) due to its possible poor objectivity. To override this problem, we aimed to analyze if an objective diagnosis of $\mathrm{HE}$ may confer additional mortality risk beyond MELD. Study and validation cohorts of patients with cirrhosis were considered in Italy and Cana$\mathrm{da}$, respectively. Patients were considered to be $\mathrm{HE}+$ if an episode of overt $\mathrm{HE}$ was documented in a hospitalization. Of the 486 patients enrolled in Italy, $184(38 \%)$ were HE+. During the 6-month follow-up, 77 patients died and 50 underwent transplantation. The 6-month mortality of $\mathrm{HE}+$ versus $\mathrm{HE}-$ patients was significantly higher $(P<0.001)$. Model for End-Stage Liver Disease (MELD; subdistribution hazard ratio [sHR], 1.2; 95\% confidence interval [CI], 1.1-1.2; $P<0.001), \mathrm{HE}+(\mathrm{sHR}$, 3.6; 95\% CI, 1.8-7.1; $P<0.001$ ), and sodium (sHR, 0.9; 95\% CI, 0.8-0.9; $P<0.001$ ) were independent predictors of 6-month mortality. In HE+ patients, short-term mortality increased across the entire MELD spectrum (range, 6-40). The results were unchanged by including or excluding patients with hepatocellular carcinoma or stratifying patients according to $\mathrm{HE}$ characteristics. The higher 6-month mortality of $\mathrm{HE}+$ versus $\mathrm{HE}-$ patients was confirmed also in the Canadian cohort $(P<0.001$; $\mathrm{n}=300,33 \% \mathrm{HE}+; 33$ died, 104 transplanted). A similar and statistically significant $\mathrm{C}$-index increase derived by the incorporation of HE in MELD was observed both in the Italian (from 0.67 to 0.75 ) and Canadian (from 0.69 to 0.74 ) cohorts. A score based on MELD plus 7 points (95\% CI, 4-10) for HE+ patients optimally predicted 6-month mortality in the 2 cohorts. According to the net reclassification index, by not considering HE, 29\% of overall patients were misclassified by MELD score. In conclusion, the incorporation of HE in MELD score might improve the listing and allocation policy in LT.
\end{abstract}

Liver Transplantation 22 1333-1342 2016 AASLD.

Received February 1, 2016; accepted June 28, 2016.

\section{SEE EDITORIAL ON PAGE 1319}

Abbreviations: $A U R O C$, area under the receiver operating characteristic curve; CFF, critical ficker frequency; CI, confidence interval; cMELD, corrected Model for End-Stage Liver Disease; EEG, electroencephalogram; $H B V$, hepatitis $B$ virus; $H C C$, hepatocellular carcinoma; HCV, hepatitis $C$ virus; $H E$, hepatic encephalopathy; HESA, Hepatic Encephalopathy Scoring Algorithm; LT, liver transplantation; MELD, Model for End-Stage Liver Disease; Na, sodium; NRI, net reclassification index; NS, not significant; ROC, receiver operating characteristic; sHR, subdistribution hazard ratio; TIPS, transjugular intrahepatic portosystemic shunt.
Liver transplantation (LT) represents the only curative treatment for patients with end-stage liver disease, and because of the continuous amelioration in immunosuppressive regimens and surgical techniques, the outcome after LT has progressively improved to up to $70 \%$ at 5 years. ${ }^{(1)}$ However, the donor pool is still scarce and many patients are not able to benefit from timely transplantation. In this scenario, the policy for the optimization of the organ allocation criteria is of great importance and is still under debate. Theoretically, the 
"ideal" method for patient prioritization would be accurate, objective, reproducible, and easy to apply.

For many years, the Child-Turcotte-Pugh score, originally designed to assess the survival of patients with cirrhosis undergoing surgery, was used for the prioritization of the patients on the waiting list for LT. ${ }^{(2)}$ However, this score has several limitations, including possible bias related to the subjective interpretation of the severity of ascites and hepatic encephalopathy (HE), and the lack of the assessment of kidney function, which represents a reliable prognostic factor in liver cirrhosis. ${ }^{(3)}$ Since 2002, the Model for End-Stage Liver Disease (MELD) score, initially developed for predicting survival in patients with cirrhosis undergoing transjugular intrahepatic portosystemic shunt (TIPS), has replaced the Child-Turcotte-Pugh score for patient prioritization. ${ }^{(4)}$ MELD is totally based on laboratory data. The introduction of MELD score for organ allocation and of the sickest-first policy for the patient prioritization has led to a significant reduction in wait-list mortality. ${ }^{(5,6)}$

Since the use of the MELD score, questions have been raised as to whether complications of liver cirrhosis provide additional prognostic information useful for LT allocation policy. ${ }^{(7)}$ To date, the MELD score is implemented with additional points only when a T2 hepatocellular carcinoma (HCC) is diagnosed to avoid disadvantaging patients with $\mathrm{HCC}$ in an allocation system exclusively based on MELD.

$\mathrm{HE}$ is a common and severe complication of cirrhosis. It is now considered not completely reversible as even a single episode of $\mathrm{HE}$ seems to be followed by permanent cognitive impairment. ${ }^{(8-11)} \mathrm{HE}$ has been related to a worse prognosis, ${ }^{(12-14)}$ and this observation was recently confirmed also in patients with acute-onchronic liver failure. ${ }^{(15)}$ Moreover, there is no correlation between the severity of $\mathrm{HE}$ and MELD score. ${ }^{(16,17)}$ Therefore, by not considering $\mathrm{HE}$ in the

Address reprint requests to Oliviero Riggio, M.D., Division of Gastroenterology, Department of Clinical Medicine, "Sapienza" University of Rome, Viale dell'Università 37, 00185 Rome, Italy. Telephone: + 39-06-4-9972001; FAX: + 11-39-6-445-3319; E-mail: oliviero.riggio@uniroma1.it

Copyright $@ 2016$ by the American Association for the Study of Liver Diseases.

View this article online at wileyonlinelibrary.com.

DOI 10.1002/lt.24517

Potential conflict of interest: Nothing to report. current allocation policy, patients with this complication might not receive LT in a timely manner, and those with HE and a MELD score below the minimum required to be listed could be wrongly excluded from LT. According to this hypothesis, a recent Italian consensus gave the opportunity to consider $\mathrm{HE}$ as an exception of MELD, assigning to this complication a fully arbitrary score (until 29) that was defined by a multidisciplinary team. ${ }^{(18)}$

Actually, a retrospective study, as well as an analysis of United Network for Organ Sharing registry data, demonstrated that grade 3-4 HE may provide additional prognostic information independent of MELD. ${ }^{(19)}$ However, HE grading is far too subjective to be easily reproducible, and criteria based on $\mathrm{HE}$ severity can hardly be shared among different centers. More recently, a model incorporating electroencephalographic parameters to MELD was proposed as a quantitative index of $\mathrm{HE}$ in the allocation policy. ${ }^{(20)}$ However, electroencephalogram (EEG) is seldom performed and accurately graded in centers dedicated to LT.

Thus, the present study was aimed at analyzing if an objective diagnosis of overt $\mathrm{HE}$, defined by a history of documented hospitalization for HE, might improve the accuracy of MELD in LT allocation policy. In particular, we studied an Italian cohort of patients with cirrhosis in order to answer 3 specific questions:

1. Is the objective diagnosis of previous overt $\mathrm{HE}$ related to a worse prognosis?

2. Is it independent of MELD score?

3. Is a MELD-HE score more accurate than MELD score alone both in listing and prioritizing patients for LT?

Moreover, in order to test the applicability of a score derived from the first cohort and including $\mathrm{HE}$ and MELD, we analyzed the relationship between HE, MELD, and short-term survival in an independent Canadian cohort of patients with cirrhosis waiting for LT.

\section{Patients and Methods}

\section{STUDY COHORT (ITALIAN PATIENTS)}

The study retrospectively analyzed demographic, clinical, and biochemical data prospectively collected in 2 groups of Italian patients with cirrhosis. The first group included all outpatients consecutively listed for primary deceased donor LT between 2002 and $2013(\mathrm{n}=301)$; 
the second one included all inpatients with cirrhosis consecutively hospitalized from 2009 to 2013 ( $n=185$ ) and systematically followed up in our outpatient services. The second group was analyzed to consider the impact of $\mathrm{HE}$ in patients with a wide MELD score range (considering also MELD $<15$ ) in order to assess the possible effect of $\mathrm{HE}$ not only in allocation but also in prioritization. Only patients aged between 18 and 70 years were included because only this age is considered for adult LT. Exclusion criteria were as follows: advanced neoplastic diseases, including $\mathrm{HCC}$ outside of Milan criteria; severe extrahepatic diseases; and concomitant neurological diseases. Patients with TIPS were also excluded because a previous study showed that in TIPS patients, the presence of $\mathrm{HE}$ was not associated with worse survival after adjusting for MELD. ${ }^{(18)}$

Demographic, clinical, and biochemical parameters were recorded at inclusion (entry to the waiting list or hospital admission) for each patient. A detailed clinical history was obtained in relation to previous complications of liver cirrhosis, and in particular to previous episodes of overt $\mathrm{HE}$. The patients were qualified as $\mathrm{HE}+$ if a previous episode of overt $\mathrm{HE} \geq$ grade 2 (according to West Haven criteria) was documented by a previous hospitalization. The cutoff for grade $2 \mathrm{HE}$ was the presence of an acute confusional syndrome with clear disorientation in time on neurological examination reported in the patient's record. HE episodes reported only by the patients or their relative during an outpatient visit were not considered enough to provide sufficient objectivity to indicate the patient as $\mathrm{HE}+$. Moreover, because of the uncertainty in the clinical staging of lowgrade $\mathrm{HE}$ (grade 1, or covert HE), in case of a degree of $\mathrm{HE}<$ grade 2, the patient was qualified as having a negative history of overt $\mathrm{HE}$ (or $\mathrm{HE}-$ ). This pragmatic definition was chosen to ensure objectivity and reproducibility in the transplant allocation setting and was defined a priori before revising the patient's record both for the Italian and the Canadian cohort.

Biological MELD score was calculated for each patient at enrollment. A corrected Model for EndStage Liver Disease (cMELD) score of 22 was assigned to the patients with HCC graded as T2 or more within Milan criteria.

All patient complications of the liver disease including $\mathrm{HE}$ were treated according to the current guidelines from the European Association for the Study of the Liver and the American Association for the Study of Liver Diseases. Patients were followed until LT, the placement of TIPS, or death.

\section{VALIDATION COHORT (CANADIAN PATIENTS)}

A second cohort of patients with cirrhosis consecutively observed and listed for LT in the Liver Unit, Division of Gastroenterology, University of Alberta, Edmonton, Canada between January 2006 and December 2009 was retrospectively analyzed as the validation group $(n=300)$. The parameters recorded, the definition of $\mathrm{HE}+$ patients, and the exclusion criteria used in the study cohort were also fully applied to the validation cohort. The study was approved by the respective institutional review boards.

\section{STATISTICAL ANALYSIS}

Patient demographics and baseline biochemical parameters were tabulated and compared using the $t$ test or nonparametric test for continuous variables, and the chi-square or Fisher's exact test for categorical variables. The MELD score was calculated using the standard formula: $11.2 \times \ln ($ international normalized ratio $)+9.57 \times \ln ($ creatinine, in $\mathrm{mg} / \mathrm{dL})+3.78 \times \ln$ (bilirubin, in $\mathrm{mg} / \mathrm{dL}$ ) +6.43 , with a lower limit of 1 for all variables.

The 2 Italian cohorts were pooled only after the demonstration of a lack of relevant differences. The conditional hazard on mortality, given that transplant is a competing risk for death, was evaluated using a proportional subdistribution hazards Fine and Gray model and a Gray test at univariate analysis. We therefore report the subdistribution hazard ratios (sHRs) rather than the classical hazard ratios, but the former have a similar interpretation to the latter.

A score was then built using predictors selected at multivariate analysis. Time-dependent receiver operating characteristic (ROC) curves $^{(21)}$ for censored data were estimated using the Kaplan-Meier estimation method; significance tests and confidence intervals (CIs) were assessed through the nonparametric bootstrap. Increases in the C-index and the net reclassification index (NRI) were used to assess improvements in discrimination after the addition of $\mathrm{HE}$ to MELD. ${ }^{(2)}$ The indexes were estimated at 3 and 6 months.

The findings were validated by computing the timedependent ROC curves on the Canadian cohort and by checking that the area under the receiver operating characteristic curve (AUROC) was not significantly different. 
Because the 2 cohorts were not significantly different in terms of AUC, in order to obtain the most precise possible estimate, we merged them when estimating the optimal number of points to add to MELD for $\mathrm{HE}+$. This was computed by maximizing the estimated $\mathrm{C}$-index at 6 months, and the 95\% CI was obtained by using the nonparametric bootstrap. For HCC patients, biological MELD is often not reflective of their mortality risk, and a cMELD is applied. We performed 2 different analyses including or excluding the patients with HCC. Moreover, in the group of patients with HCC, we considered both the biological MELD and the cMELD with exception for HCC. Data were analyzed using R, version 3.0.2 (R Foundation, Vienna, Austria).

\section{Results}

\section{STUDY COHORT}

A total of 311 cirrhotic outpatients listed for LT were considered: 10 patients were excluded for previous TIPS placement. A second group of 235 hospitalized patients with cirrhosis not listed for LT was also analyzed: from this group, 17 patients were excluded for the presence of advanced neoplastic diseases, 19 for the diagnosis of severe comorbidities, and 14 for the presence of TIPS.

The cohort of the patients not listed for LT was considered to have a larger MELD spectrum. This variability allowed us to analyze the capacity of the new criterion (HE) not only for prioritization but also for entry into the waiting list. This second important aim could not be possible if we had only considered the patients already included on a waiting list.

The follow-up of these 2 groups was identical. The 2 groups of outpatients $(\mathrm{n}=301)$ and inpatients $(\mathrm{n}=185)$ were similar for age $(55 \pm 8.5$ versus $57 \pm 9$ years; not significant [NS]), sex (males, $72 \%$ versus $74 \%$; NS), severity of liver disease (MELD, $15 \pm 5$ versus $14.5 \pm 6$; NS), degree of portal hypertension as clinically represented by presence of gastroesophageal varices (64\% versus 59\%; NS), and history of ascites ( $48 \%$ versus $53 \%$; NS). The most common causes of liver disease were also similar in both groups: viral (45\% versus $42 \%$; NS), alcoholic ( $23 \%$ versus $26 \%$; NS), and viral + alcoholic (12\% versus $12.7 \%$; NS). Outpatients were more often affected by HCC than inpatients (39\% versus 26\%; $P<0.01$ ). The 2 groups
TABLE 1. Demographic, Clinical, and Biochemical Characteristics at the Enrollment of Study Cohort (Italian) and Validation Cohort (Canadian)

\begin{tabular}{|c|c|c|c|}
\hline Variables & $\begin{array}{c}\text { Study } \\
\text { Cohort } \\
(n=475)\end{array}$ & $\begin{array}{c}\text { Validation } \\
\text { Cohort } \\
(n=300)\end{array}$ & $P$ Values \\
\hline Age, years & $55.4 \pm 9.9$ & $53 \pm 8.6$ & 0.02 \\
\hline Sex, males & $343(72)$ & $208(69)$ & 0.30 \\
\hline \multicolumn{4}{|l|}{ Main origin of liver disease } \\
\hline Alcohol & $158(33)$ & $98(33)$ & 0.80 \\
\hline $\mathrm{HCV}$ & $144(30)$ & $93(31)$ & \\
\hline HBV & 59 (12) & $27(9)$ & \\
\hline Viral + alcohol & $59(12)$ & $35(12)$ & \\
\hline Gastroesophageal varices & $294(62)$ & $155(52)$ & 0.01 \\
\hline Ascites & 235 (49) & $227(76)$ & $<0.001$ \\
\hline $\mathrm{HCC}$ & $162(34)$ & $76(25)$ & 0.01 \\
\hline Diabetes & $130(27)$ & $53(18)$ & $<0.01$ \\
\hline Organ renal failure & $47(10)$ & $13(4)$ & $<0.01$ \\
\hline Biological MELD score & $15.5(6-40)$ & $17(9-40)$ & $<0.01$ \\
\hline Serum sodium, mEq/L & $136 \pm 5.1$ & $135.7 \pm 5.1$ & 0.40 \\
\hline
\end{tabular}

NOTE: Data are given as $\mathrm{n}(\%)$ or mean \pm standard deviation.

were then pooled and analyzed as a whole group (Table 1).

The HE+ group included 189 (40\%) patients. The characteristics of patients with and without $\mathrm{HE}$ are reported in Table 2. Patients who were $\mathrm{HE}+$ were slightly older than those who were HE-, but other demographic characteristics, such as causes of cirrhosis and comorbidities, did not differ significantly between the 2 groups. Liver disease was more severe in the patients with $\mathrm{HE}$, as indicated by MELD score, MELD-Na score, history of ascites, and biochemical parameters. The prevalence of $\mathrm{HCC}$ was similar. According to the diagnostic definition, all $\mathrm{HE}+$ patients had been previously hospitalized at least once, but also those without $\mathrm{HE}$ exhibited a very high rate of hospitalizations ( $80 \%$ versus $100 \% ; P<0.001$; Table 2). In $\mathrm{HE}+$ patients, the MELD score at the time of the HE episode was similar to the MELD score calculated at enrollment $(14.9 \pm 7.3$ versus $15.7 \pm 5.6$; $P=0.24)$.

During 6-month follow-up, 77 (16\%) patients died, $50(11 \%)$ underwent transplantation, and $6(1.5 \%)$ had undergone a TIPS; 21 (4\%) patients were lost to follow-up. As reported in Fig. 1, the 6-month mortality rate of the $\mathrm{HE}+$ patients was significantly higher than that of $\mathrm{HE}$ - patients. This result was observed both in the entire population (Fig. 1A) and when the patients with HCC were excluded (Fig. 1B) to consider the biological MELD only.

A number of clinical and biochemical variables potentially associated with short-term mortality were submitted to a univariate and multivariate analysis. 
TABLE 2. Demographic, Clinical, and Biochemical Characteristics of the Patients According to the Presence of HE in the Study Cohort

\begin{tabular}{|c|c|c|c|}
\hline Variables & $\begin{array}{c}\text { HE- Patients } \\
(n=286)\end{array}$ & $\begin{array}{c}\mathrm{HE}+\text { Patients } \\
(\mathrm{n}=189)\end{array}$ & $P$ Values \\
\hline Age, years & $55 \pm 9.9$ & $57 \pm 9.7$ & 0.045 \\
\hline Sex, males & $208(73)$ & $135(71)$ & 0.80 \\
\hline \multicolumn{4}{|l|}{ Origin of liver disease } \\
\hline Alcohol & $74(26)$ & $45(24)$ & \multirow[t]{5}{*}{0.55} \\
\hline $\mathrm{HCV}$ & $82(29)$ & $64(34)$ & \\
\hline HBV & 46 (16) & $13(7)$ & \\
\hline Viral + alcohol & $34(12)$ & $25(13)$ & \\
\hline Other & $50(17)$ & $42(22)$ & \\
\hline Gastroesophageal varices & $173(60)$ & $121(64)$ & 0.50 \\
\hline Ascites & $120(42)$ & $117(62)$ & $<0.001$ \\
\hline $\mathrm{HCC}$ & $106(37)$ & $56(30)$ & 0.09 \\
\hline Diabetes & $70(24)$ & $60(32)$ & 0.20 \\
\hline Organ renal failure & $26(9)$ & $21(11)$ & 0.50 \\
\hline MELD score & $14 \pm 5.1$ & $15.7 \pm 5.6$ & $<0.001$ \\
\hline MELD $\geq 15$ & $67(23)$ & $58(31)$ & 0.07 \\
\hline MELD-Na score & $15.8 \pm 6$ & $19 \pm 6.6$ & $<0.001$ \\
\hline Serum creatinine, $\mathrm{mg} / \mathrm{dL}$ & $1.0 \pm 0.6$ & $1.1 \pm 0.9$ & 0.09 \\
\hline Serum albumin, $\mathrm{g} / \mathrm{dL}$ & $3.3 \pm 0.6$ & $3.1 \pm 0.6$ & $<0.001$ \\
\hline International normalized ratio & $1.4 \pm 0.3$ & $1.5 \pm 0.4$ & $<0.001$ \\
\hline Serum bilirubin, $\mathrm{mg} / \mathrm{dL}$ & $3.5 \pm 5$ & $4.3 \pm 6$ & 0.10 \\
\hline Platelets, $\mathrm{n} / \mathrm{mm}^{3}$ & $212,000 \pm 108,000$ & $160,000 \pm 60,000$ & 0.30 \\
\hline Serum sodium, mEq/L & $137 \pm 4.8$ & $134.5 \pm 6$ & $<0.001$ \\
\hline Actual or previous hospitalization for any reasons & $229(80)$ & $189(100)$ & $<0.001$ \\
\hline Patients dead in the first 6 months, $\mathrm{n}$ & 14 & 63 & $<0.001$ \\
\hline \multicolumn{4}{|l|}{ Causes of death } \\
\hline Liver failure & $3(21)$ & $11(17)$ & \multirow[t]{4}{*}{0.30} \\
\hline Infections & $5(36)$ & $31(49)$ & \\
\hline Variceal bleeding & $2(14)$ & $8(13)$ & \\
\hline Other & $4(29)$ & $13(21)$ & \\
\hline
\end{tabular}

NOTE: Data are given as $\mathrm{n}(\%)$ or mean \pm standard deviation.

$\mathrm{HE}+$ status, MELD score, serum sodium levels, the presence or history of ascites, and hospitalizations (actual or previous) for any reasons were significantly associated with both 3- and 6-month mortality (Table 3).

When the above parameters were included in a multivariate analysis, $\mathrm{HE}+(\mathrm{sHR}, 3.6$; 95\% CI, 1.8-7.1; $P<0.001$ ), MELD (sHR, 1.2; 95\% CI, 1.1-1.2; $P<0.001$ ), and serum sodium levels (sHR, 0.9; 95\% CI, 0.8-0.9; $P<0.001)$ were independently associated with 6-month mortality. Similar results were obtained when 3-month mortality was considered (data not shown). A MELD score of $>15$ is commonly used as a criterion for listing the patients for LT. When only the patients with a MELD score of $>15$ were considered, the presence of $\mathrm{HE}$ was associated with a 3.2fold increase in 6-month mortality risk $(95 \% \mathrm{CI}, 1.7$ 6; $P<0.001$ ), suggesting that $\mathrm{HE}$ could be a factor for prioritizing patients already listed for LT. Also in patients with MELD $<15$, the 6 -month mortality risk was increased 5.6-fold by $\mathrm{HE}$ (95\% CI, 1.6-20; $P<0.01)$, suggesting that a history of $\mathrm{HE}$ should be taken into consideration for listing patients for LT even if the MELD score does not allow it.

The $189 \mathrm{HE}+$ patients were further classified according to the following: maximum severity of $\mathrm{HE}$ reported in the hospital records (grade 2 versus grade 3-4); HE time course (episodic versus recurrent defined as bouts of $\mathrm{HE}$ occurring with a time interval of 6 months or less); and proximity between the first episode of $\mathrm{HE}$ and enrollment ( $>6$ or $\leq 6$ months). The 1-year mortality was similar in these subgroups (Table 4), suggesting that a history of overt $\mathrm{HE}$ is the main prognostic factor and that a further characterization of the $\mathrm{HE}$ episodes in terms of severity, time course, and distance is not needed.

\section{VALIDATION COHORT}

The validation group consisted of 300 patients consecutively listed for LT in the Liver Unit, Division of Gastroenterology, University of Alberta, Edmonton, Canada. Demographic and clinical characteristics of these patients are reported in Table 1 . The patients 

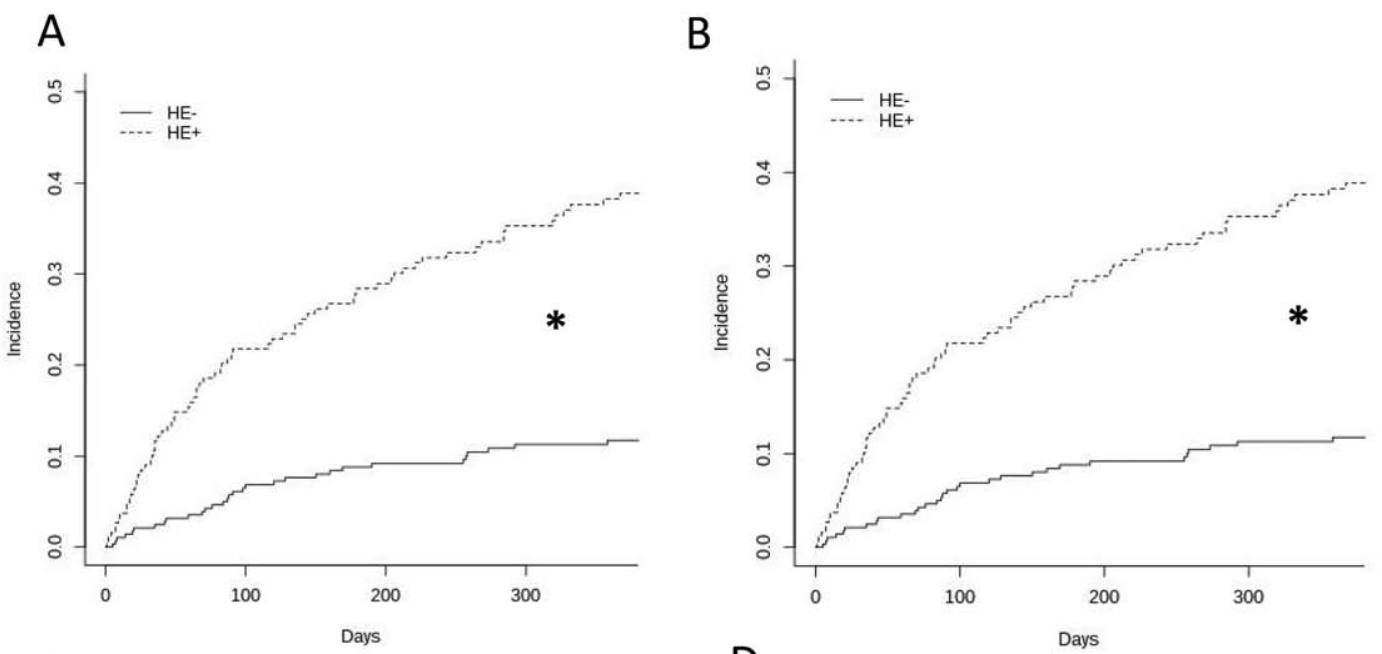

C

D
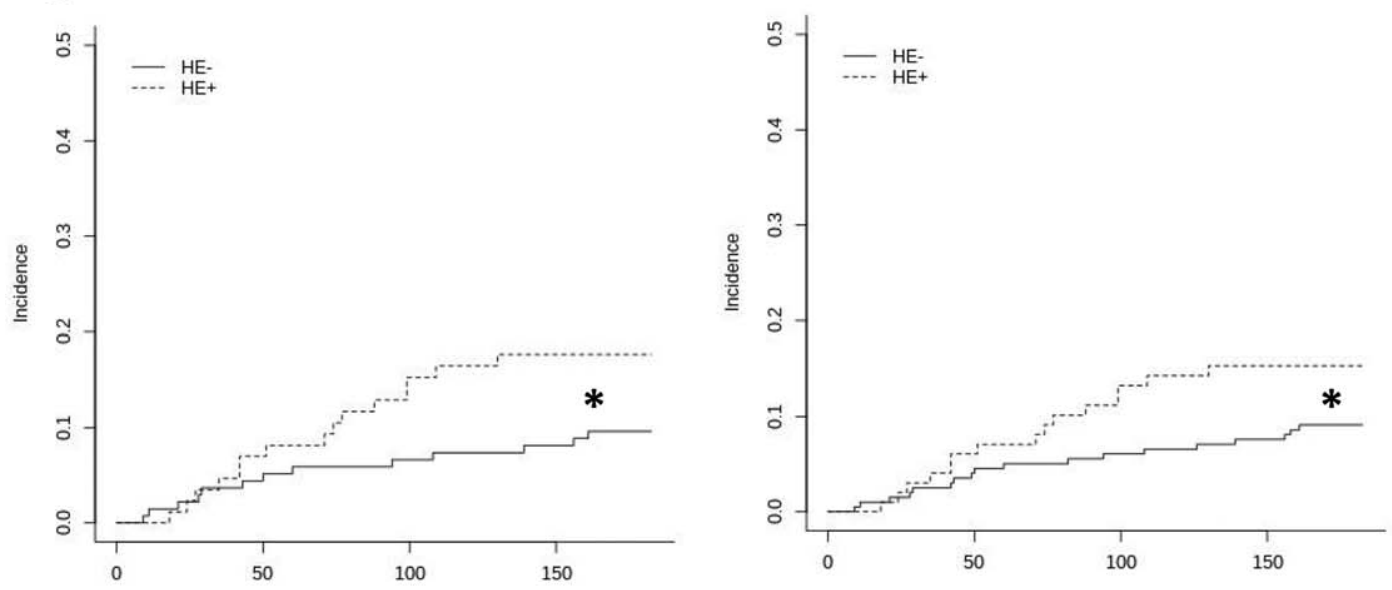

\section{$\mathrm{E}$}
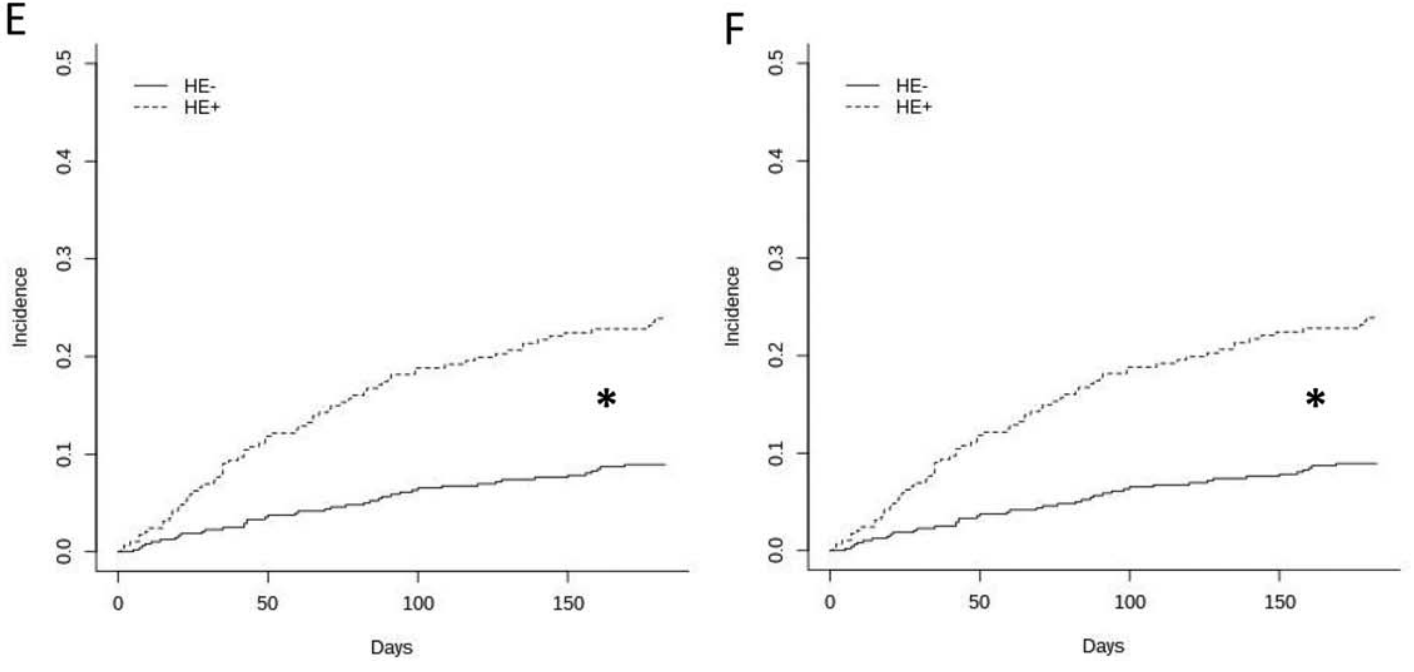

FIG. 1. Cumulative 6-month mortality rate is shown according to HE status in (A) the study cohort, (C) the validation cohort, and (E) in the combination of the 2 cohorts. (B), (D), and (F) show the cumulative mortality rate in the same cohorts, respectively, excluding the patients with HCC. ${ }^{*} P<0.01$. 
TABLE 3. Univariate Analysis of Factors Associated With 3- and 6-Month Mortality in the Study Cohort

\begin{tabular}{|c|c|c|c|c|c|c|}
\hline & \multicolumn{3}{|c|}{3 Months } & \multicolumn{3}{|c|}{6 Months } \\
\hline & sHR & $95 \% \mathrm{Cl}$ & $P$ Values & sHR & $95 \% \mathrm{Cl}$ & $P$ Values \\
\hline MELD score & 1.1 & $1.1-1.2$ & $<0.001$ & 1.16 & $1.1-1.21$ & $<0.001$ \\
\hline Serum sodium & 0.84 & $0.79-0.89$ & $<0.001$ & 0.85 & $0.81-0.90$ & $<0.001$ \\
\hline $\mathrm{HE}+$ & 4.53 & $2.45-8.37$ & $<0.001$ & 4.25 & $2.52-7.19$ & $<0.001$ \\
\hline $\mathrm{HCC}$ & 0.55 & $0.29-1.05$ & 0.07 & 0.63 & $0.37-1.10$ & 0.10 \\
\hline Ascites & 2.20 & $1.30-3.73$ & $<0.01$ & 2.54 & $1.54-4.20$ & $<0.001$ \\
\hline Hospitalization for any reasons & 8.66 & $1.18-63.80$ & 0.03 & 12.70 & $1.73-93.15$ & 0.01 \\
\hline Age & 1.01 & $0.98-1.04$ & 0.60 & 1.01 & $0.98-1.03$ & 0.53 \\
\hline Sex, male & 1.27 & $0.66-2.44$ & 0.48 & 1.52 & $0.84-2.76$ & 0.16 \\
\hline Alcoholic origin & 0.57 & $0.30-1.10$ & 0.09 & 0.71 & $0.42-1.23$ & 0.22 \\
\hline
\end{tabular}

included in the validation group were slightly younger and were affected by a more severe disease than the patients included in the study cohort (higher MELD score and higher prevalence of ascites) although with a lower prevalence of gastroesophageal varices. The prevalence of HCC was also lower. At the time of LT, the MELD scores were $18.4 \pm 4.5$ in the Italian cohort and $17.5 \pm 5$ in the Canadian cohort. According to the definition of overt HE used in the study cohort, 33\% of patients included in the validation cohort were classified as $\mathrm{HE}+$. During 6 months of follow-up, 33 patients died and 104 underwent transplantation.

As reported in Fig. 1C, the 6-month mortality rate of the $\mathrm{HE}+$ patients was significantly higher than that of $\mathrm{HE}-$ patients $(P<0.001)$. This result was observed also when the patients with $\mathrm{HCC}$ were excluded (Fig. 1D).

The AUCs of MELD alone in the 6-month mortality prediction obtained in the Italian and Canadian cohorts were not statistically different $(0.67$ and 0.69 , respectively; $P=0.70$ ).

The added value of $\mathrm{HE}+$ status to a score consisting only of MELD was confirmed also in this cohort through a significant $\mathrm{C}$-index increase (from 0.69 to $0.74 ; P=0.04)$ similar to that obtained in the study

TABLE 4. One-Year Mortality in the Patients With HE Stratified According to HE Characteristics in Term of Severity, Time Course, and Timing of the First Episode of HE

\begin{tabular}{llll} 
& $\begin{array}{c}\text { Dead } \\
(\mathrm{n}=90)\end{array}$ & $\begin{array}{c}\text { Alive } \\
(\mathrm{n}=99)\end{array}$ & P Values \\
\hline HE severity, $\mathrm{n}(\%)$ & & & \\
$\quad$ Grade 2 & $49(54.4)$ & $58(58.6)$ & 0.50 \\
$\quad$ Grade 3-4 & $41(45.6)$ & $41(41.1)$ & \\
HE time course, $\mathrm{n}(\%)$ & $44(49)$ & $63(63.6)$ & 0.10 \\
$\quad$ Episodic & $46(51)$ & $36(36.4)$ & \\
$\quad$ Recurrent & & & \\
$\begin{array}{l}\text { Timing of the first episode of HE } \\
\leq 6 \text { months }\end{array}$ & $22(24)$ & $13(13.1)$ & 0.10 \\
$\quad 66$ months & $68(76)$ & $86(86.9)$ &
\end{tabular}

group $(P=0.36)$. In all cases, 7 points were added to the MELD scores of $\mathrm{HE}+$ patients (see below).

\section{COMBINED ANALYSIS OF THE 2 COHORTS (STUDY AND VALIDATION)}

After the demonstration that the 2 cohorts were not different in terms of C-index of MELD plus HE, the data were combined in order to obtain a score by a wider number of patients.

The mortality rate observed in the combined series of patients according to $\mathrm{HE}$ status is reported in Fig. $1 \mathrm{E}, \mathrm{F}$.

Figure 2 reports the 6-month mortality rate by MELD score, stratified by HE status and estimated through the Fine and Gray competing risk regression

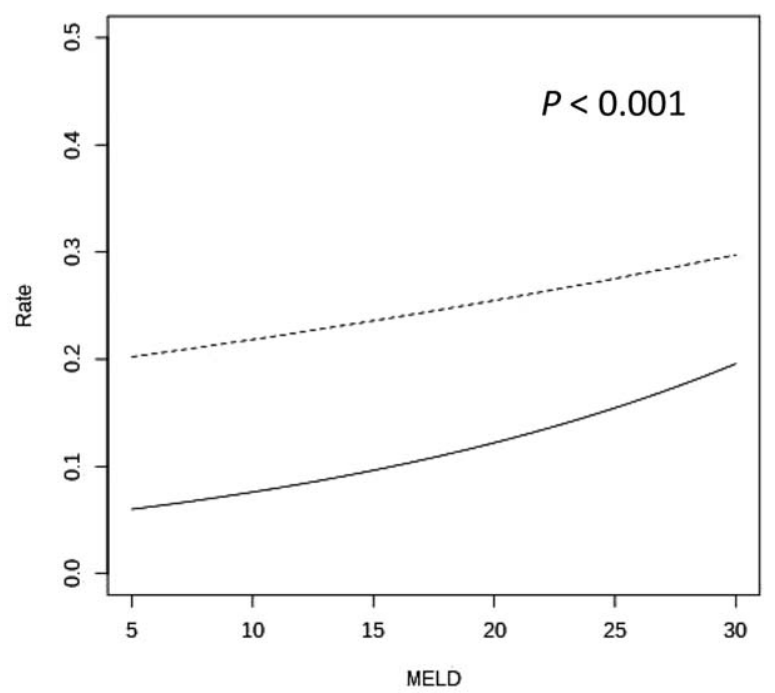

FIG. 2. Overall study (2 cohorts) estimated 6-month mortality rate by MELD score in patients with $\mathrm{HE}+$ (dotted line) and $\mathrm{HE}-$ (continuous line). 
model. It shows that mortality increases across the entire MELD spectrum in $\mathrm{HE}+$ patients. The optimal number of points to be added to the MELD scores of patients with $\mathrm{HE}$ was estimated by the AUROC for 3- and 6-month mortality. A significant increase in $\mathrm{C}$-index (from 0.73 to $0.78 ; P<0.01$ ) in predicting 6-month mortality was obtained by incorporating $\mathrm{HE}$ into MELD. The value obtained by maximizing the AUROC was 7, indicating that a score based on MELD +7 points (95\% CI, 4-10) for $\mathrm{HE}+$ patients optimally predicts the 6-month mortality rate. The NRI, as a measure to assess improvements in discrimination after the addition of $\mathrm{HE}$, was 29\% (95\% CI, 19.4-41.4; $P<0.01$ ), suggesting that this percentage of patients is misclassified by MELD alone and may be correctly classified taking into account overt HE. Similar results were obtained when the 3-month mortality rate was considered: $\mathrm{C}$-index improved from 0.74 to $0.79(P=0.01)$ and NRI was $31 \%(95 \% \mathrm{CI}$, $17.5-40.4 ; P<0.001)$. Finally, these results were observed both in the entire population and when the patients with HCC were excluded (data not shown).

\section{Discussion}

The prioritization of LT for end-stage liver disease patients uses the MELD score in order to identify the sickest patients as those in greatest need for LT. Since the introduction of MELD, questions have been raised as to whether complications of liver cirrhosis provide additional prognostic information useful for LT allocation policy. ${ }^{(7)} \mathrm{HE}$ is one of the most frequent complications of liver cirrhosis, and since the Child-Pugh era, it has been known to be related to a worse prognosis. ${ }^{(12-14)}$ This relationship was recently confirmed even in patients with acute-on-chronic liver failure. ${ }^{(15)}$ Thus, our hypothesis was that in patients with $\mathrm{HE}$, the severity of liver disease may be underestimated by MELD alone, and we aimed to offer a practical and pragmatic method to include HE in MELD score. For this aim, we adopted a definition of HE not based on severity in order to avoid the misclassification bias and subjectivity. In fact, in our opinion, despite the strong evidence of its prognostic role, $\mathrm{HE}$ is currently not considered for listing and prioritizing the patients for LT, mainly because of the subjectivity in its assessment and staging. Our pragmatic definition based only on the presence of a clear episode of $\mathrm{HE}$ (grade 2 or higher) documented in a patient's record was chosen to guarantee as much objectivity as possible. According to these criteria, $\mathrm{HE}$ documented in a outpatient record only or referred to by the patients or their relatives was not considered sufficient to classify the patients as $\mathrm{HE}+$.

We tried to address this issue by reviewing the data prospectively collected in patients seen in Italy. We also considered patients with cirrhosis included and not included on the waiting list for LT in our analysis to test the capacity of $\mathrm{HE}+$ status in the selection of candidates for the waiting list.

The results were then validated by comparing the AUC of the model relating MELD and HE with 6month survival derived from the Italian cohort with that obtained from the Canadian cohort. It should be stressed that the 2 cohorts included patients seen in far different countries and characterized by statistically significant differences in demographic and clinical data (Table 1). A validation in 2 so different cohorts supports the applicability of the model based on MELD and $\mathrm{HE}$ in very different settings. Given these results, the 2 cohorts were merged to obtain an estimation of the relationship between MELD without $\mathrm{HE}$ and survival based on a large series of patients. In this way, the range of MELD scores in the whole series was quite large (range, 6-40), allowing us to analyze the impact of $\mathrm{HE}$ across the whole MELD spectrum. Moreover, a sensitivity analysis was carried out in order to determine the applicability of these results to patients with HCC in whom exception MELD points are given. The results clearly showed that MELD alone underestimated the short-term mortality of patients with a previous episode of $\mathrm{HE}$ and suggested that doctors should take into consideration this important complication of liver cirrhosis in LT allocation policy. The problem is the introduction of a clinical parameter into a score exclusively based on numerical data. In fact, the main advantages of MELD are that it is entirely objective, reproducible, and easy to apply because it is completely based on laboratory data. In contrast, the diagnosis of overt $\mathrm{HE}$ as well as of the other complications of cirrhosis may be operator-dependent. Even the numerical scores proposed for HE staging (Hepatic Encephalopathy Scoring Algorithm [HESA] score or New Haven score) are based on subjective judgments of the patient's state of consciousness or behavior and are not easily reproducible in the different centers competing for organ allocation. Psychometric tests (computerized or "paper and pencil") are able to provide numerical parameters, but they can be used only for compliant enough patients. ${ }^{(23-25)}$ The same is true for the critical flicker frequency $(\mathrm{CFF})$, which was recently 
proposed as a parameter useful to explore the cognitive impairment of patients with cirrhosis related to the patient's survival. ${ }^{(26)}$ More importantly, once it is realized that wait-list inclusion may depend on the result of these tests, a patient may voluntary alter his or her psychometric performance. For these reasons, HE was until now not considered in allocation scores for LT, despite the abundant evidence of its prognostic relevance. We tried to solve this problem using a pragmatic definition of $\mathrm{HE}$ by considering $\mathrm{HE}+$ patients to be those with a documented diagnosis of overt HE made during a hospitalization. This "user-friendly" method goes over the need of classifying the patients according to $\mathrm{HE}$ severity (because of the possible subjectivity) and to other characteristics. In fact, the maximum severity of $\mathrm{HE}$ reported in the hospital records (grade 2 versus grade 3-4), HE time course (episodic versus recurrent defined as bouts of $\mathrm{HE}$ occurring with a time interval of 6 months or less), and proximity between the first episode of $\mathrm{HE}$ and enrollment ( $>$ or $\leq$ of 6 months) did not influence the 6-month mortality, probably also because of the higher difficulty in recording these data. The choice to consider only HE episodes documented by a previous hospitalization strongly reduces the subjectivity in $\mathrm{HE}$ diagnosis.

Our analysis shows the following:

1. HE, as defined above, significantly worsens the short-term prognosis independent of MELD and the other complications of liver cirrhosis.

2. In patients with a documented history of $\mathrm{HE}$, short-term mortality increases across the entire MELD spectrum, suggesting that $\mathrm{HE}$ is a factor both for listing and prioritizing patients for LT.

3. A score based on MELD +7 points $(95 \% \mathrm{CI}$, 4-10) for $\mathrm{HE}+$ patients optimally predicts the 6-month mortality rate.

4. By not considering $\mathrm{HE}$, about a third of the patients are misclassified by MELD alone in terms of short-term mortality.

Before our study, at least 4 articles underlined the importance of HE for LT allocation. Stewart et al., ${ }^{(19)}$ by analyzing the mortality rate of 271 hospitalized patients, showed that $\mathrm{HE}$ grade 2 or higher was associated with an increased mortality independently on MELD. Wong et al., ${ }^{(14)}$ using the United Network for Organ Sharing registry data, evaluated the impact of $\mathrm{HE}$ on 90-day wait-list survival in almost 90,000 patients with cirrhosis, showing that patients with grade 3-4 $\mathrm{HE}$ had a $66 \%$ greater risk of 90-day mortality than patients without $\mathrm{HE}$ and that when stratified by MELD, patients with grade 3-4 HE had a 90-day wait-list mortality similar to nonencephalopathic patients with MELD scores 6-7 points higher. Montagnese et al., ${ }^{(20)}$ using EEG and automated determination of its mean dominant frequency in 392 patients with cirrhosis, showed that both MELD and EEG were independent predictors of mortality and that MELDEEG had higher prognostic accuracy in predicting 12and 18-month mortality compared to MELD. Finally, Ampuero et al. ${ }^{(26)}$ showed that CFF was related to patient survival in patients with a MELD score higher than 10 .

Although prospective studies are still needed, our results support the idea of considering $\mathrm{HE}$ together with MELD in the current LT allocation policy (for inclusion and prioritization of patients) without using difficult tools and trying to propose a method of quantification of $\mathrm{HE}$ prognostic role. Although MELD alone with the exclusion of $\mathrm{HE}$ among the staging criteria causes a third of patients to be misclassified in terms of short-term mortality, the optimal correction of MELD score to take into consideration the influence of $\mathrm{HE}$ would be the addition of 7 points in patients with a documented episode of overt HE. Considering $\mathrm{HE}$ among the criteria for LT may also be useful for assessing timing and urgency for bedside clinicians to pursue alternative means to transplant (ie, living donor or extended criteria grafts).

\section{REFERENCES}

1) Adam R, Hoti E. Liver transplantation: the current situation. Semin Liver Dis 2009;29:3-18.

2) Child CG, Turcotte JG. Surgery and portal hypertension. Major Probl Clin Surg 1964;1:1-85.

3) Forman LM, Lucey MR. Predicting the prognosis of chronic liver disease: an evolution from child to MELD. Mayo End-stage Liver Disease. Hepatology 2001;33:473-475.

4) Kamath PS, Wiesner RH, Malinchoc M, Kremers W, Therneau TM, Kosberg CL, et al. A model to predict survival in patients with end-stage liver disease. Hepatology 2001;33:464-470.

5) Freeman RB, Wiesner RH, Edwards E, Harper A, Merion R, Wolfe R; for United Network for Organ Sharing Organ Procurement and Transplantation NetworkLiver and Transplantation Committee. Results of the first year of the new liver allocation plan. Liver Transpl 2004;10:7-15.

6) Fink MA, Angus PW, Gow PJ, Berry SR, Wang BZ, Muralidharan V, et al. Liver transplant recipient selection: MELD vs. clinical judgment. Liver Transpl 2005;11:621-626.

7) Bernardi M, Gitto S, Biselli M. The MELD score in patients awaiting liver transplant: strengths and weaknesses. J Hepatol 2011;54:1297-1306.

8) Bajaj JS, Schubert CM, Heuman DM, Wade JB, Gibson DP, Topaz A, et al. Persistence of cognitive impairment after resolution of overt hepatic encephalopathy. Gastroenterology 2010;138: 2332-2340.

9) Riggio O, Ridola L, Pasquale C, Nardelli S, Pentassuglio I, Moscucci F, Merli M. Evidence of persistent cognitive 
impairment after resolution of overt hepatic encephalopathy. Clin Gastroenterol Hepatol 2011;9:181-183.

10) Umapathy S, Dhiman RK, Grover S, Duseja A, Chawla YK. Persistence of cognitive impairment after resolution of overt hepatic encephalopathy. Am J Gastroenterol 2014;109:10111019.

11) Campagna F, Montagnese S, Schiff S, Biancardi A, Mapelli D, Angeli $\mathrm{P}$, et al. Cognitive impairment and electroencephalographic alterations before and after liver transplantation: what is reversible? Liver Transpl 2014;20:977-986.

12) Bustamante J, Rimola A, Ventura PJ, Navasa M, Cirera I, Reggiardo V, Rodés J. Prognostic significance of hepatic encephalopathy in patients with cirrhosis. J Hepatol 1999;30:890-895.

13) Said A, Williams J, Holden J, Remington P, Gangnon R, Musat A, Lucey MR. Model for end stage liver disease score predicts mortality across a broad spectrum of liver disease. J Hepatol 2004;40:897-903.

14) Wong RJ, Gish RG, Ahmed A. Hepatic encephalopathy is associated with significantly increased mortality among patients awaiting liver transplantation. Liver Transpl 2014;20:1454-1461.

15) Cordoba J, Ventura-Cots M, Simón-Talero M, Amorós À, Pavesi M, Vilstrup H, et al.; for CANONIC Study Investigators of EASL-CLIF Consortium. Characteristics, risk factors, and mortality of cirrhotic patients hospitalized for hepatic encephalopathy with and without acute-on-chronic liver failure (ACLF). J Hepatol 2014;60:275-281.

16) Yoo HY, Edwin D, Thuluvath PJ. Relationship of the model for end-stage liver disease (MELD) scale to hepatic encephalopathy, as defined by electroencephalography and neuropsychometric testing, and ascites. Am J Gastroenterol 2003;98:13951399.
17) Bajaj JS, Saeian K. MELD score does not discriminate against patients with hepatic encephalopathy. Dig Dis Sci 2005;50:753-756.

18) Cillo U, Burra P, Mazzaferro V, Belli L, Pinna AD, Spada M, et al.; for I-BELT (Italian Board of Experts in the Field of Liver Transplantation). A multistep, consensus-based approach to organ allocation in liver transplantation: toward a "blended principle model." Am J Transplant 2015;15:2552-2561.

19) Stewart CA, Malinchoc M, Kim WR, Kamath PS. Hepatic encephalopathy as a predictor of survival in patients with endstage liver disease. Liver Transpl 2007;13:1366-1371.

20) Montagnese S, De Rui M, Schiff S, Ceranto E, Valenti P, Angeli P, et al. Prognostic benefit of the addition of a quantitative index of hepatic encephalopathy to the MELD score: the MELD-EEG. Liver Int 2015;35:58-64.

21) Heagerty PJ, Lumley T, Pepe MS. Time-dependent ROC curves for censored survival data and a diagnostic marker. Biometrics 2000;56:337-344.

22) Pencina MJ, D'Agostino RB Sr, Steyerberg EW. Extensions of net reclassification improvement calculations to measure usefulness of new biomarkers. Stat Med 2011;30:11-21.

23) Kircheis G, Fleig WE, Görtelmeyer R, Grafe S, Häussinger D. Assessment of low-grade hepatic encephalopathy: a critical analysis. J Hepatol 2007;47:642-650.

24) Montagnese S, Schiff S, Turco M, Bonato CA, Ridola L, Gatta A, et al. Simple tools for complex syndromes: a three-level difficulty test for hepatic encephalopathy. Dig Liver Dis 2012;44:957-960.

25) Montagnese S, Schiff S, De Rui M, Crossey MM, Amodio P, Taylor-Robinson SD. Neuropsychological tools in hepatology: a survival guide for the clinician. J Viral Hepat 2012;19:307-315.

26) Ampuero J, Simón M, Montoliú C, Jover R, Serra MÁ, Córdoba J, Romero-Gómez M. Minimal hepatic encephalopathy and critical flicker frequency are associated with survival of patients with cirrhosis. Gastroenterology 2015;149:1483-1489. 